\title{
ANALYTIC CONTINUATION FROM A FAMILY OF LINES
}

\author{
Alexander Tumanov
}

\begin{abstract}
Given a function $f$ in the exterior of a convex curve in the real plane, we prove that if the restrictions of $f$ to the tangent lines to the curve extend as entire functions, then the function $f$ is an entire function of two variables.
\end{abstract}

We prove the following

Theorem 1. Let $\Lambda \subset \mathbf{R}^{2}$ be a $C^{2}$-smooth convex curve with strictly positive curvature. Let $f$ be a complex function in the exterior of $\Lambda$. Denote by $l_{\lambda}$ the tangent line to $\Lambda$ at $\lambda \in \Lambda$. Suppose for every $\lambda \in \Lambda$, there is an entire function $f_{\lambda}$ on $\mathbf{C}=\mathbf{R}^{2}$ such that the restrictions of $f$ and $f_{\lambda}$ to $l_{\lambda}$ coincide, that is, $\left.f\right|_{l_{\lambda}}=\left.f_{\lambda}\right|_{l_{\lambda}}$. Suppose that the map $(z, \lambda) \mapsto f_{\lambda}(z)$ is continuous. Then $f$ extends as an entire function on $\mathbf{C}^{2}$.

The condition that $\Lambda$ has positive curvature is not essential. We add it for simplicity and convenience of presentation. Instead of assuming that the map $(z, \lambda) \mapsto f_{\lambda}(z)$ is continuous, we can assume that it is locally bounded. If $\Lambda$ is real-analytic, then this condition can be dropped (Theorem 6). The author does not know whether it can be dropped if $\Lambda$ is merely smooth.

In the case $\Lambda$ is the unit circle, the result was obtained by Aguilar, Ehrenpreis and Kuchment $[\mathrm{AEK}]$ as the characterization of the range of a version of the Radon transform. Öktem [Ö] gave another proof using a separate analyticity result of Siciak [S2]. Ehrenpreis informed the author that he was able to prove Theorem 1 for algebraic $\Lambda$. The proofs in [AEK, Ö] don't go through for an arbitrary convex curve $\Lambda$. Our method is similar to that of $[\mathrm{T}]$. It is based on Lewy's [L] proof of the classical extension theorem of Kneser [K] and Lewy $[\mathrm{L}]$.

The author thanks Peter Kuchment for formulating the problem and useful discussions. The author thanks Guiseppe Zampieri for useful discussions. 
Turning to the proof, we introduce

$$
\begin{aligned}
& \Sigma=\left\{(z, w) \in \mathbf{C}^{2}: w=\bar{z}\right\} \\
& h_{\lambda}=\left\{(z, \bar{z}): z \in l_{\lambda}\right\} \subset \Sigma
\end{aligned}
$$

Let $L_{\lambda}$ be the complex line in $\mathbf{C}^{2}$ containing the real line $h_{\lambda}$. Define a hypersurface

$$
M=\bigcup_{\lambda \in \Lambda} L_{\lambda} .
$$

Define a function $F$ on $M$ that consists of all the extensions $f_{\lambda}$ of the original function $f$, that is,

$$
F(z, w)=f_{\lambda}(z) \quad \text { if } \quad(z, w) \in L_{\lambda} .
$$

We will show that $F$ extends as an entire function on $\mathbf{C}^{2}$. Then the original function $f$ extends as an entire function of $z$ and $\bar{z}$, hence, as an entire function of $\operatorname{Re} z$ and $\operatorname{Im} z$ as desired.

We first describe the geometry of $M$. Let $\Lambda_{i}$ and $\Lambda_{e}$ denote the two connected components of $\mathbf{C} \backslash \Lambda$, the interior and exterior of $\Lambda$. Suppose $0 \in \Lambda_{i}$. Likewise, we put

$$
\Pi=\{(z, w) \in \Sigma: z \in \Lambda\}, \quad \Pi_{e}=\left\{(z, w) \in \Sigma: z \in \Lambda_{e}\right\} .
$$

Since $\Lambda$ has positive curvature, the line $L_{\lambda}$ moves transversally to itself as $\lambda$ traces $\Lambda$ except at $\Pi$, where the $L_{\lambda}$ is sliding along itself. Then $M \backslash \Pi$ is a smooth immersed hypersurface. The lines $L_{\lambda}$ intersect only on $\Pi_{e}$, and every point of $\Pi_{e}$ is the point of intersection of exactly two lines $L_{\lambda}$. Hence, $M \backslash \Pi$ is a real Levi-flat hypersurface in $\mathbf{C}^{2}$ with transverse self-intersection on $\Pi_{e}$.

We parametrize $\Lambda$ by the arc length $s \mapsto \lambda \in \Lambda$ in counterclockwise direction, then $\lambda^{\prime}=d \lambda / d s$ is the unit tangent vector at $\lambda$, in particular $\left|\lambda^{\prime}\right|=1$. The tangent line $l_{\lambda}$ has the parametric equation

$$
z=\lambda+t \lambda^{\prime}, \quad t \in \mathbf{R} .
$$

Then $h_{\lambda} \subset \Sigma$ has the parametric equations

$$
z=\lambda+t \lambda^{\prime}, \quad w=\bar{\lambda}+t \bar{\lambda}^{\prime}, \quad t \in \mathbf{R} .
$$

Eliminating $t$ we get

$$
\frac{z-\lambda}{\lambda^{\prime}}=\frac{w-\bar{\lambda}}{\bar{\lambda}^{\prime}} \in \mathbf{R}
$$


Then the complexification $L_{\lambda}$ of $h_{\lambda}$ has the equation

$$
\frac{z-\lambda}{\lambda^{\prime}}=\frac{w-\bar{\lambda}}{\bar{\lambda}^{\prime}}
$$

Let $l_{\lambda}^{ \pm}$be the two components of $\mathbf{C} \backslash l_{\lambda}$, with $0 \in l_{\lambda}^{+}$, that is,

$$
l_{\lambda}^{ \pm}=\left\{z \in \mathbf{C}: \pm \operatorname{Im} \frac{z-\lambda}{\lambda^{\prime}}>0\right\}
$$

We put

$$
L_{\lambda}^{ \pm}=\left\{(z, w) \in L_{\lambda}: z \in l_{\lambda}^{ \pm}\right\}, \quad M^{ \pm}=\bigcup_{\lambda \in \Lambda} L_{\lambda}^{ \pm} .
$$

Then $M \backslash \Sigma=M^{+} \cup M^{-}$. We study the geometry of $M$ by looking at the intersections with coordinate lines

$$
\Gamma_{z}=\{w \in \mathbf{C}:(z, w) \in M\}, \quad \Gamma_{z}^{ \pm}=\left\{w \in \mathbf{C}:(z, w) \in M^{ \pm}\right\}
$$

where $z \in \mathbf{C}$ is fixed. The curve $\Gamma_{z}$ is parametrized as

$$
\Lambda \ni \lambda \mapsto w(\lambda)=\bar{\lambda}+\left(\bar{\lambda}^{\prime}\right)^{2}(z-\lambda)
$$

Note that for $\lambda_{1} \neq \lambda_{2}, L_{\lambda_{1}} \cap L_{\lambda_{2}}=h_{\lambda_{1}} \cap h_{\lambda_{2}} \subset \Sigma$. Then $\Gamma_{z}$ can have self-intersection only at the point $w=\bar{z}$. The point $(z, \bar{z}) \in h_{\lambda}$ if and only if $\frac{z-\lambda}{\lambda^{\prime}} \in \mathbf{R}$, that is, $z \in l_{\lambda}$.

If $z \in \Lambda_{i}$, then for every $\lambda \in \Lambda$, we have $\operatorname{Im} \frac{z-\lambda}{\lambda^{\prime}}>0$, hence $\Gamma_{z}=\Gamma_{z}^{+}$is a simple closed curve and $\Gamma_{z}^{-}=\emptyset$.

If $z \in \Lambda_{e}$, then there are two tangent lines $l_{\lambda}$ through $z$. The points of tangency split $\Lambda$ into two arcs

$$
\Lambda^{ \pm}(z)=\left\{\lambda \in \Lambda: w(\lambda) \in \Gamma_{z}^{ \pm}\right\}=\left\{\lambda \in \Lambda: \pm \operatorname{Im} \frac{z-\lambda}{\lambda^{\prime}}>0\right\}
$$

The $\operatorname{arc} \Lambda^{-}(z)$ is the one closer to $z$. Hence, the curve $\Gamma_{z}$ consists of two simple disjoint loops $\Gamma_{z}^{ \pm}$initiating and terminating at $\bar{z}$.

We claim that $\Gamma_{z}^{-}$lies inside $\Gamma_{z}^{+}$. Indeed, we have

$$
\left|w(\lambda)-\left(\bar{\lambda}^{\prime}\right)^{2} z\right|=\left|\bar{\lambda}-\lambda\left(\bar{\lambda}^{\prime}\right)^{2}\right| \leq 2|\lambda| \leq 2 m, \quad \text { where } \quad m=\max _{\lambda \in \Lambda}|\lambda| .
$$

Denote by ind $\phi$ the index or winding number of a map $\phi: \Lambda \rightarrow \mathbf{C}$ around 0 as $\Lambda$ is traced counterclockwise. Then ind $\lambda^{\prime}=1$, ind $\left(\bar{\lambda}^{\prime}\right)^{2} z=-2$. In view of the above estimate of $w(\lambda)$, 
for big $|z|$, we have ind $\Gamma_{z}=-2$. This implies that $\Gamma_{z}^{ \pm}$lie inside one another. The curve $\Gamma_{z}^{-}$contracts into $\bar{z}_{0}$ as $\Lambda_{e} \ni z \rightarrow z_{0} \in \Lambda$. Hence, $\Gamma_{z}^{-}$is the inner one.

Let $D_{z}^{+}, D_{z}^{-}$, and $D_{z}^{0}$ be the three connected components of $\mathbf{C} \backslash \Gamma_{z}$ : the exterior of $\Gamma_{z}^{+}$, the interior of $\Gamma_{z}^{-}$, and the domain between them. If $z \in \Lambda_{i}$, then $D_{z}^{-}=\emptyset$. Then

$$
\Omega^{\nu}=\bigcup_{z \in \mathbf{C}}\{z\} \times D_{z}^{\nu}, \quad \nu=+,-, 0
$$

are the three connected components of $\mathbf{C}^{2} \backslash M$. The estimate of $w(\lambda)$ implies $D_{z}^{ \pm} \supset\{w \in$ $\mathbf{C}: \pm(|w|-|z|)>2 m\}$, hence,

$$
\Omega^{ \pm} \supset\left\{(z, w) \in \mathbf{C}^{2}: \pm(|w|-|z|)>2 m\right\} .
$$

By the hypotheses of Theorem 1, the function $F$ defined above is a continuous CR function on $M$.

Lemma 2. $F$ holomorphically extends into $\Omega^{-}$.

Proof. We follow the proof of the H. Lewy [L] extension theorem. We define the extension $\tilde{F}$ by the Cauchy type integral

$$
\tilde{F}(z, w)=\frac{1}{2 \pi i} \int_{\Gamma_{z}^{-}} \frac{F(z, \zeta) d \zeta}{\zeta-w}, \quad z \in \Lambda_{e}, \quad w \in D_{z}^{-} .
$$

Obviously, $\tilde{F}$ is holomorphic in $w$. To see that $\tilde{F}$ is an extension of $F$, we prove the vanishing of the moments

$$
m_{n}(z)=\int_{\Gamma_{z}^{-}} \zeta^{n} F(z, \zeta) d \zeta, \quad z \in \Lambda_{e}, \quad n \geq 0
$$

We first show that both $\tilde{F}$ and $m_{n}$ are holomorphic in $z$. Put $\Phi(z)=\int_{\Gamma_{z}^{-}} H(z, \zeta) d \zeta$, where $H$ is the integrand in the formulas for $\tilde{F}$ and $m_{n}$. By the Morera theorem, it suffices to show that $\int_{\gamma} \Phi(z) d z=0$ for every small loop $\gamma$ in $\Lambda_{e}$.

The torus $T=\bigcup_{z \in \gamma}\{z\} \times \Gamma_{z}^{-} \subset M$ bounds a solid torus $S \subset M$ obtained by filling the loop $\gamma$. Then by Stokes' formula

$$
\int_{\gamma} \Phi(z) d z=\int_{T} H(z, \zeta) d \zeta \wedge d z=\int_{S} d H(z, \zeta) \wedge d \zeta \wedge d z=0
$$

because $H$ is a CR function on $M$. 
Recall that $\Gamma_{z}^{-}$contracts into $\bar{z}_{0}$ as $\Lambda_{e} \ni z \rightarrow z_{0} \in \Lambda$. Then $m_{n}(z) \rightarrow 0$ as $z \rightarrow z_{0}$. By the boundary uniqueness theorem, $m_{n}(z)=0$ identically. By the moment condition, $\tilde{F}$ is the holomorphic extension of $F$ as desired. The lemma is proved.

Note that the interchange $z \leftrightarrow w$ leads to the interchange $\Omega^{+} \leftrightarrow \Omega^{-}$. Then $F$ also extends into $\Omega^{+}$. It remains to prove

Lemma 3. $\tilde{F}$ holomorphically extends into $\Omega^{0}$.

Proof. Let

$$
X_{c}=\{(z, w): z w=c\},
$$

where $c \in \mathbf{C}$, and $|c|$ is large. The intersection $K_{c}=X_{c} \cap M$ is (the image of) the map $K_{c}: \Lambda \ni \lambda \mapsto X_{c} \cap L_{\lambda}$. The curve $K_{c}$ can have self-intersection only if $w=\bar{z}$, hence $c=|z|^{2}>0$.

We use $z$ as a coordinate on $X_{c}$, which identifies $X_{c}$ with the punctured complex plane $\mathbf{C} \backslash\{0\}$. Solving the equations for $X_{c} \cap L_{\lambda}$, we find

$$
z=\frac{-i b \pm \sqrt{c-b^{2}}}{\bar{\lambda}^{\prime}}, \quad b=\operatorname{Im}\left(\lambda^{\prime} \bar{\lambda}\right)
$$

If $c \notin \mathbf{R}$, then $K_{c}$ consists of two disjoint loops corresponding to the two values of the square root. They are different because $K_{c}$ can't have self-intersections.

Since $|b| \leq m$, then both components of $K_{c}$ are within fixed distance from the circle $|z|=\sqrt{|c|}$. Hence they lie inside one another. Let $X_{c}^{+}, X_{c}^{-}$, and $X_{c}^{0}$ be the three components of $X_{c} \backslash K_{c}$, with $X_{c}^{+}$being the inmost component, $X_{c}^{-}$being the outmost component, and $X_{c}^{0}$ being the annulus between them. Then $X_{c}^{ \pm} \subset \Omega^{ \pm}$because $\Omega^{ \pm} \supset$ $\{(z, w) \in \mathbf{C}: \pm(|w|-|z|)>2 m\}$. Hence $X_{c}^{0} \subset \Omega^{0}$.

If $c \in \mathbf{R}^{+}$, the positive reals, then the two curves corresponding to the two values of the square root coincide and have the surprisingly simple form

$$
K_{c}=\{(z, w):|z|=\sqrt{c}, w=\bar{z}\} .
$$

We now use the same argument as in Lemma 2 in the coordinate system $(z, c=z w)$. We define the extension $\tilde{F}$ to $X_{c}^{0}$ by the Cauchy integral along $K_{c}$. The vanishing of the moments will take place because the integrals along the two components of $K_{c}$ will cancel in the limit as $\mathbf{C} \backslash \mathbf{R}^{+} \ni c \rightarrow c_{0} \in \mathbf{R}^{+}$. This will yield the holomorphic extension of $F$ to $X_{c}$ for large $c$. Then by the Hartogs extension theorem, $\tilde{F}$ extends to the whole space $\mathbf{C}^{2}$.

Lemma 3 and Theorem 1 are now proved. 
In conclusion, we show how to get rid of the continuity hypothesis in Theorem 1 if $\Lambda$ is real-analytic. We use a separate analyticity result by Siciak [S1].

Lemma 4. (Siciak [S1]) Let $E_{\nu} \subset \mathbf{C}(\nu=1,2)$ be the domain bounded by the ellipse with foci $\pm r_{\nu}$, and let $I_{\nu}=\left[-r_{\nu}, r_{\nu}\right] \subset \mathbf{R}$. Let $X=\left(E_{1} \times I_{2}\right) \cup\left(I_{1} \times E_{2}\right) \subset \mathbf{C}^{2}$. Let $f: X \rightarrow \mathbf{C}$ be separately holomorphic, that is, for every $x_{1} \in I_{1}, f\left(x_{1}, \cdot\right)$ is holomorphic in $E_{2}$, and for every $x_{2} \in I_{2}, f\left(\cdot, x_{2}\right)$ is holomorphic in $E_{1}$. Then $f$ holomorphically extends into a neighborhood of $X$.

Bernstein (1912) proved Lemma 4 under an additional boundedness assumption. He gave a precise description of the domain of the extension, but we don't need it here. Lemma 5 implies a propagation of analyticity result for separately holomorphic functions.

Proposition 5. Let $M$ be a real-analytic Levi-flat hypersurface in $\mathbf{C}^{2}$. Let $S \subset M$ be a connected complex curve. Let $F: M \rightarrow \mathbf{C}$ be a separately holomorphic function, that is, $F$ is holomorphic on complex curves in $M$. Suppose $F$ holomorphically extends to a neighborhood of $p \in S$ in $\mathbf{C}^{2}$. Then $F$ holomorphically extends to a neighborhood of the whole curve $S$ in $\mathbf{C}^{2}$.

Proof. Without loss of generality, in a local coordinate system $z=\left(z_{1}, z_{2}\right)=x+i y$, $M$ and $S$ have the equations $x_{2}=0$ and $z_{2}=0$ respectively, and $p=(0,0)$. Choose $r_{1}=r_{2}$ and $E_{2}$ so small that $F$ is holomorphic in a neighborhood of $I_{1} \times E_{2}$. Then by Lemma $5, F$ extends to a neighborhood of $I_{2} \times E_{1}$ for big $E_{1}$. The proposition is proved.

Theorem 6. Let $\Lambda \subset \mathbf{R}^{2}$ be a real-analytic convex curve with strictly positive curvature. Let $f$ be a complex function in the exterior of $\Lambda$. Let $l_{\lambda}$ be the tangent line to $\Lambda$ at $\lambda \in \Lambda$. Suppose for every $\lambda \in \Lambda$, there is an entire function $f_{\lambda}$ on $\mathbf{C}=\mathbf{R}^{2}$ such that $\left.f\right|_{l_{\lambda}}=\left.f_{\lambda}\right|_{l_{\lambda}}$. Then $f$ extends as an entire function on $\mathbf{C}^{2}$.

Proof. We need to show that if $\Lambda$ is real-analytic, then the function $F$ in the proof of Theorem 1 is continuous on $M$. We first show that $F$ is real-analytic on $\Pi_{e} \subset \Sigma$. Following [Ö], we define

$$
\phi: Y=(\mathbf{R} \times \mathbf{R}) \backslash K \rightarrow \Pi_{e}, \quad \phi\left(s_{1}, s_{2}\right)=h_{\lambda\left(s_{1}\right)} \cap h_{\lambda\left(s_{2}\right)},
$$

where $K$ is the set of all $\left(s_{1}, s_{2}\right)$ such that the lines $h_{\lambda\left(s_{1}\right)}$ and $h_{\lambda\left(s_{2}\right)}$ are parallel. Since $\Lambda$ is real-analytic, then so is $\phi$. Then $\phi$ extends to a holomorphic map $\Phi$ in a neighborhood of $Y$ in $\mathbf{C}^{2}$. Let $p \in \Pi_{e}, p=\phi\left(c_{1}, c_{2}\right)$. We use $\Phi^{-1}$ to introduce local coordinates $\left(s_{1}+i t_{1}, s_{2}+i t_{2}\right)$ in a neighborhood of $p$. In these coordinates $M$ is represented as the 
union of the hyperplanes $s_{1}=c_{1}$ and $s_{2}=c_{2}$, on which $F$ is separately holomorphic. By Lemma 4 the function $F$ holomorpically extends to a neighborhood of $p$. Finally, by Proposition 5, the analyticity of $F$ propagates along complex lines in $M$, so $F$ is realanalytic, whence continuous on $M$. The theorem is proved.

\section{References}

[AEK] V. Aguilar, L. Ehrenpreis, P. Kuchment, Range condition for the exponential Radon transform, J. d'Analyse Math. 68 (1996), 1-13.

[K] H. Kneser, Die Randwerte einer analytischen Funktion zweier Vernderlichen, Monatsh. Math. Phys. 43 (1936), 364-380.

[L] H. Lewy, On the local character of the solutions of an atypical linear differential equation in three variables and a related theorem for regular functions of two complex variables, Ann. Math. 64 (1956), 514-522.

[Ö] O. Öktem, Extension of separately analytic functions and applications to range characterization of the exponential Radon transform, Ann. Polon. Math. 70 (1998), 195-213.

[S1] J. Siciak, Analyticity and separate analyticity of functions defined on lower dimensional subsets of $\mathbf{C}^{n}$, Zeszyty Nauk. Uniw. Jagiello. Prace Mat. Zeszyt 13 (1969), 53-70.

[S2] J. Siciak, Separately analytic functions and envelopes of holomorphy of some lower dimensional subsets of $C^{n}$, Ann. Polon. Math. 22 (1969/1970), 145-171.

[T] A. Tumanov, Testing analyticity on circles, Amer. J. Math. 129 (2007), 785-790.

\section{Alexander Tumanov}

Department of Mathematics, University of Illinois

1409 W. Green Street, Urbana, IL 61801, U. S. A.

E-mail: tumanov@uiuc.edu 